\title{
Clinico- Biochemical Profile of Neonatal Seizure.
}

Shah GS ${ }^{1}$, Singh MK ${ }^{2}$, Budhathoki $\mathrm{S}^{3}$, Kalakheti BK ${ }^{4}$, Baral DD ${ }^{5}$

1. Dr. Gauri Shankar Shah, Assistant Professor, ${ }^{2 .}$ Dr. Manoj Kumar Singh, Senior Resident, ${ }^{3 .}$ Dr. Sunil Budhathoki, Senior Resident, ${ }^{4}$. Dr. Balkrishna Kalakheti, Assistant Professor, Department of Pediatrics and Adolescent Medicine, ${ }^{5 \cdot}$ Mr. DD Baral. Assistant Professor, Biostatistics, B P Koirala Institute of Health Sciences, Dharan, Nepal.

Address for correspondence: gaurisshah@yahoo.com

\begin{abstract}
Objectives: The present study was undertaken to study the incidence, etiological factor, and days of onset, clinical types and biochemical abnormalities in babies having neonatal seizures. Materials and Methods: This is a retrospective, observational hospital based study. Ninety neonates, who developed seizures before 28 days of life and admitted to neonatal intensive care unit and nursery with neonatal seizure, were evaluated for incidence, etiological factor, clinical types and biochemical abnormalities found in neonatal seizure. The variables were analysed using Chi- Square and student t- test. Results: The incidence of neonatal seizure was 10.3/ 1000 live births. The seizures were common in male babies. $65(72.2 \%)$ neonates were born to multiparous women while $35(38 \%)$ were born to primigravidas. In birth asphyxia $(n=40)$, the most common type of seizure observed was subtle seizure $20(50 \%)$, followed by focal clonic 10 (25\%) and multifocal clonic 5 (12.5\%). Tonic type of seizure was observed in $3(7.5 \%)$ and myoclonic in $2(5 \%)$. Meningitis and septicaemia was the second most common cause of neonatal seizure observed in our study. Among metabolic abnormalities hypoglycaemia was found in $20(22 \%)$ and hypocalcaemia in $10(11 \%)$. Conclusion: The commonest cause of seizure was birth asphyxia presenting within 72 hours of life. Among infection septicaemia and meningitis was the most common cause leading to neonatal seizure. Among biochemical abnormalities the most common cause of seizure observed in our study was hypoglycaemia and hypocalcaemia. Subtle seizures were the commonest type of seizure observed in this study.
\end{abstract}

Key words: Neonatal seizure, biochemical disturbances in seizure.

\section{Introduction:}

The presence of seizure does not constitute a diagnoses but it is a symptom of an underlying central nervous system disorder due to systemic or biochemical disturbances. Biochemical disturbances occur frequently in the neonatal seizures either as an underlying cause or as an associated abnormality. In their presence, it is difficult to control seizure and there is a risk of further brain damage. Early recognition and treatment of biochemical disturbances is essential for optimal management and satisfactory long-term outcome. Seizures represent the signal of neurological disease in the newborn period and these convulsive phenomena are the most frequent manifestation of neonatal neurological disorder ${ }^{1}$. Delay in recognition and treatment results in poor neurological outcome $^{2}$. Seizures during the neonatal period are relatively common, occurring in 1.8 to 3.5 per 1000 live births, with greater frequency in premature or low birth weight babies as compared to term babies ${ }^{3}$. Neonatal seizures are always due to an underlying cerebral or biochemical abnormalities although no cause may be identified in $10 \%$ of cases ${ }^{4}$. In the Neonatal Intensive Care Unit, the incidence goes as high as $10-25 \%$ out of which about $15 \%$ will die and 35 to $40 \%$ will have major neurological sequelae ${ }^{5}$. There is increasing evidence that neonatal seizures have an adverse effect on neurodevelopment and may predispose to cognitive, behavioural or epileptic complication later in life ${ }^{6}$. The present study was conducted to evaluate the incidence, etiological factor, clinical types and to observe various biochemical abnormalities.

\section{Materials and Methods:}

The present study was a retrospective, observational study conducted in the Neonatal Intensive Care Unit and Nursery at B. P. Koirala Institute of Health Sciences from $1^{\text {st }}$ December 2006 to $15^{\text {th }}$ October 2007. All neonates with seizure before 28 days of life were included in the study. The neonatal seizures were classified according to Volpe's classification into subtle, focal clonic, multifocal clonic, tonic and myoclonic. Metabolic abnormalities and infections were noted. Hypoglycemia were defined as blood sugar $<40 \mathrm{mg} / \mathrm{dl}$, and hypocalcaemia when total serum calcium was less than 7.0 $\mathrm{mg} / \mathrm{dl}$. Total 90 cases of neonatal seizure presenting before 28 days of life was included in the study. Age, sex, etiological factors and biochemical parameters were recorded in a predesigned data sheet. The data was analysed by using SPSS 10.0 version.

\section{Results:}

Total admission during the study period was 867 live births, out of which 90 neonates presented with seizure. The incidence of neonatal seizure was $10.3 / 1000$ live births. The seizures were more common in male babies observed in our study. $65(72.2 \%)$ neonates were born to multiparous women 
while $35(38 \%)$ were born to primigravidas. In birth asphyxia $(n=40)$, the most common type of seizure observed was subtle seizure $20(50 \%)$, followed by focal clonic 10 (25\%) and multifocal clonic $5(12.5 \%)$. Tonic type of seizure was observed in $3(7.5 \%)$ and myoclonic in $2(5 \%)$. Meningitis and septicaemia was the second most common cause of neonatal seizure observed in our study. Among metabolic abnormalities hypoglycaemia was found in 20 (22\%) and hypocalcaemia in $10(11 \%)$. The commonest cause of seizure was birth asphyxia presenting within 72 hours. Among infection septicaemia and meningitis was the most common infection leading to neonatal seizure. Among biochemical abnormalities the most common cause of seizure observed in our study was hypoglycaemia and hypocalcaemia. Subtle seizures were the commonest type of seizure observed in this study. In majority of cases the seizure occurred within 24 hours that has been shown in Table III.

Table 1: Incidence of Neonatal Seizure According to Weight.

\begin{tabular}{|l|c|c|c|}
\hline Weight of the neonates & $\begin{array}{c}\text { Total no. } \\
\text { neonates }\end{array}$ & $\begin{array}{c}\text { Neonates } \\
\text { with seizure }\end{array}$ & Percentage \\
\hline$<2500$ gms (LBW) & 400 & 60 & $66 \%$ \\
\hline 2500 gms & 467 & 30 & $33 \%$ \\
\hline
\end{tabular}

et al who reported 5\% developed septicaemia and $12 \%$ developed meningitis ${ }^{\mathbf{1 0}}$. In present study hypocalcaemia is observed in $11 \%$ and hypoglycaemia in $20 \%$. The study conducted by Kumar A et al ${ }^{11}$ found that birth asphyxia was the commonest cause of seizure in first $48 \mathrm{hrs}$ of life which was similar to our finding. In the present study prolonged rupture of membrane, Meconium stained liquor/ foul smelling liquor, repeated vaginal examination during pregnancy, repeated instrumentation, maternal fever, maternal diarrhoea and prematurity were the risk factors for development of neonatal sepsis. The presence of seizure does not constitute a diagnosis but it is a symptom of an underlying central nervous system disorder due to systemic or biochemical disturbances. Hypoglycaemia and hypocalcaemia are the most common biochemical abnormality seen in neonates with seizure and carries a good short term outcome.

\section{Conclusion:}

To conclude, birth asphyxia was the commonest cause of neonatal seizure that can be managed by providing intensive care unit and by resuscitation. Early recognition and treatment will improve the long-term outcome. Meningitis and septicaemia also contributed to neonatal seizure, which needs aggressive management. Among the biochemical abnormalities

Table II: Relationship of Aetiology and Type of Seizure.

\begin{tabular}{|l|l|l|l|l|l|l|}
\hline Aetiology & $\begin{array}{l}\text { Total } \\
(\mathrm{n}=90)\end{array}$ & $\begin{array}{l}\text { Subtle } \\
(\mathrm{n}=38)\end{array}$ & $\begin{array}{l}\text { Focal clonic } \\
(\mathrm{no}=30)\end{array}$ & $\begin{array}{l}\text { Multifocal clonic } \\
(\mathrm{no}=10)\end{array}$ & $\begin{array}{l}\text { Tonic } \\
(\mathrm{no}=10)\end{array}$ & $\begin{array}{l}\text { Myoclonic } \\
(\mathrm{no}=2)\end{array}$ \\
\hline Birth asphyxia & $40(44 \%)$ & $20(50 \%)$ & $10(25 \%)$ & $5(12.5 \%)$ & $3(7.5 \%)$ & $2(5 \%)$ \\
\hline Septicaemia & $10(11 \%)$ & $5(50 \%)$ & $2(20 \%)$ & $1(10 \%)$ & $1(10 \%)$ & $1(10 \%)$ \\
\hline Meningitis & $10(11 \%)$ & $6(60 \%)$ & $1(10 \%)$ & $1(10 \%)$ & $1(10 \%)$ & $1(10 \%)$ \\
\hline Hypocalcaemia & $10(11 \%)$ & $7(70 \%)$ & $1(10 \%)$ & 0 & $1(10 \%)$ & $1(10 \%)$ \\
\hline Hypoglycaemia & $20(22 \%)$ & $13(65 \%)$ & $5(25 \%)$ & 0 & $1(5 \%)$ & $1(5 \%)$ \\
\hline
\end{tabular}

Table III: Aetiology Versus onset of seizure.

\begin{tabular}{|l|l|l|l|l|l|}
\hline Aetiology & No of cases & $0-1$ days & $1-2$ days & $3-7$ days & $<7$ days \\
\hline Birth asphyxia & 40 & $26(65 \%)$ & $12(30 \%)$ & $2(5 \%)$ & 0 \\
\hline Septicaemia & 10 & $1(10 \%)$ & $5(50 \%)$ & $1(10 \%)$ & $3(30 \%)$ \\
\hline Meningitis & 10 & $6(60 \%)$ & $2(20 \%)$ & $1(10 \%)$ & $1(10 \%)$ \\
\hline Hypocalcaemia & 10 & $7(70 \%)$ & $1(10 \%)$ & $1(10 \%)$ & $1(10 \%)$ \\
\hline Hypoglycemia & 20 & $16(80 \%)$ & $1(5 \%)$ & $2(10 \%)$ & $1(5 \%)$ \\
\hline
\end{tabular}

\section{Discussion:}

Neonatal seizure is an important cause of neonatal morbidity and mortality. The present study showed incidence of 10.3/ 1000 live births which is similar to Holden KR et al study $^{7}$. In the present study the most common type of seizure observed were subtle which constitutes $50 \%$ of seizure in both term and preterm babies ${ }^{8}$. The most common cause of seizure encountered in this study was birth asphyxia, which is comparable with Sood A et al study that reported $45.7 \%$ cases of seizure were due to birth asphyxia ${ }^{9}$. Our study showed $20 \%$ of the seizure were due to infections i.e. septicaemia and meningitis, which is also similar to study, conducted by Ledigo hypoglycaemia and hypocalcaemia was the commonest cause for neonatal seizure. Subtle seizure was the commonest type of seizure that can be easily missed, as it is very mild.

\section{References:}

1. Neonatal Seizures. In: Volpe J J eds., Neurology of the new born 4th ed. Philadelphia: W.B. Saunders, 2001:178-216.

2. Scher MS. Controversies Regarding The Neonatal Seizures Recognition Epileptic Disorders. June 2002; 4 (2): 139-158. 
3. Mizrahi Eli M. Neonatal Seizures and Neonatal Epilieptic Syndromes. Neurologic Clinics In Epilepsy. 2001; 19 (2): 427-456.

4. Neurological disorders. In: Singh M. Textbook Of Care Of New Born 5th ed., New Delhi: Sagar publication, 1999: 340-344.

5. Pleasure David, Daryl C. De Vivo. The Nervous System. In: Rudolph Textbook Of Pediatric. Colin D. Rudolph, Abraham D., Margaret K., Hostellter, 21st ed., New York: McGraw Hills, 2002: 2267.

6. Levene M. Clinical Conundrum Of Neonatal Seizures. Arch dis child fetal neonatal 2002; 86 (2): 75-77.

7. Holden KR, Mellitus D and Freeman JM. Neonatal Seizures: Correlation of Prenatal and Perinatal Events with Outcome. Pediatrics. 1982; 70: 165-176.
8. Du Pliessis AJ. Neonatal Seizures. In: Cloherty John P. Eichenwald EC and stark AR eds., Manual of neonatal care 5th ed., Philadelphia: Lippincott Williams \& Wilkins, 2004: 507-522.

9. Sood A, Grower N and Sharma R. Biochemical Abnormalities in Neonatal Seizure. Indian J Pediatric ; 70 (3): 221-224.

10. Ronen GM, Penney S and Andrew W. The Epidemiology of Clinical Neonatal Seizures in New Foundland - A Population Based Study. J. Pediatric. 1999; 134: 71-75.

11. Kumar A, Gupta V, Kacchawaha and Singla. A Study of Biochemical Abnormalities in Neonatal Seizure. Indian Pediatrics. 1995; 52: 424-427. 